\title{
CINÉTICA DA OXIDAÇÃO DE BIODIESEL DE ÓLEO DE SOJA EM MISTURA COM TBHQ: DETERMINAÇÃO DO TEMPO DE ESTOCAGEM
}

\author{
Dionísio Borsato*, Elaine Cristina R. Maia, Luiz Henrique Dall’Antonia, Hágata Cremasco da Silva e Jaqueline Laís \\ Pereira \\ Departamento de Química, Universidade Estadual de Londrina, CP 6001, 86051-990 Londrina - PR, Brasil
}

Recebido em 30/5/11; aceito em 3/11/11; publicado na web em 23/1/12

\begin{abstract}
KINETICS OF OXIDATION OF BIODIESEL FROM SOYBEAN OIL MIXED WITH TBHQ: DETERMINATION OF STORAGE TIME. Synthetic antioxidants are an alternative to prevent or retard the degradation of biofuels made from vegetable oils. In this study, it was evaluated the oxidative stability of B100 soybean oil biodiesel, in the presence of tercbutylhydroquinone (TBHQ). The results showed that the induction period, that precedes the seeding process, was delayed in the presence of the antioxidant. Moreover, the obtained results suggest that the B100 biodiesel containing TBHQ can present a storage time at $25^{\circ} \mathrm{C}$, three times longer than the estimated time for the pure B100.
\end{abstract}

Keywords: induction time; antioxidant; activation energy.

\section{INTRODUÇÃO}

O biodiesel é uma denominação genérica para combustíveis derivados de fontes renováveis, tais como óleos vegetais e gordura animal, sendo biodegradável. Possui características similares ao diesel de petróleo, apresenta a vantagem de reduzir a emissão de compostos de enxofre durante sua combustão, possui ponto de fulgor mais alto, o que lhe confere manuseio e armazenamento seguros e apresenta boa lubricidade. ${ }^{1,2}$

Ao contrário dos combustíveis fósseis que são relativamente inertes e mantêm as suas características essenciais pouco alteradas, o biodiesel degrada-se com o tempo devido ao seu contato com contaminantes, tanto de natureza inorgânica, quanto microbiana, sendo a oxidação decorrente da sua exposição ao ar atmosférico um dos principais problemas a que está sujeito. ${ }^{3}$

A estabilidade oxidativa vem sendo bastante investigada pela comunidade científica e algumas oleaginosas, fontes para produção de biodiesel, são portadoras de características químicas indesejadas, as quais são incorporadas pelo biodiesel durante o processo de obtenção. Alguns exemplos são as oleaginosas, como a soja, que apresentam uma significativa quantidade de ácidos graxos com alto grau de insaturação, que facilitam a oxidação do biocombustível, dificultando a armazenagem do mesmo. ${ }^{1}$ Portanto, como medida preventiva, é importante manter os tanques de armazenamento no limite máximo permitido, reduzindo assim a quantidade de ar em contato com o combustível. Além disso, deve estar isento de contaminantes, secos e protegidos da luz e de temperaturas extremas. ${ }^{1,4}$

Com a finalidade de inibir ou retardar a oxidação lipídica do biodiesel B100 são empregados compostos químicos conhecidos como antioxidantes e/ou estabilizadores. ${ }^{5}$

Os antioxidantes ocorrem naturalmente em óleos vegetais e os mais comuns são os tocoferóis. No entanto, alguns processos de produção de óleos vegetais incluem uma etapa de destilação para purificação dos mono-alquil ésteres. O biodiesel obtido a partir destes óleos normalmente possui pouco ou nada de antioxidantes naturais, assim, torna-se menos estável. ${ }^{6}$ Surge então a necessidade de se aplicar antioxidantes sintéticos para aumentar a estabilidade do biocombustível e, assim, manter suas propriedades por um período maior. ${ }^{4}$

*e-mail: dborsato@uel.br
O uso de antioxidantes e seus mecanismos funcionais têm sido amplamente estudados e podem ser classificados em primários, sinergistas, removedores de oxigênio, biológicos, agentes quelantes e antioxidantes mistos. Os antioxidantes primários são compostos fenólicos que promovem a remoção ou inativação dos radicais livres formados durante a iniciação ou propagação da reação, interrompendo a reação em cadeia. ${ }^{7}$

Entre os principais e mais conhecidos antioxidantes primários incluem-se o butil-hidroxianisol (BHA), butil-hidroxitolueno (BHT), terc-butil-hidroquinona (TBHQ) e propil galato (PG). A estrutura fenólica destes compostos permite a doação de um hidrogênio ao radical livre, regenerando, assim, a molécula do éster e interrompendo o mecanismo de oxidação por radicais livres. ${ }^{5}$ Dessa maneira, os derivados fenólicos transformam-se em radicais livres, entretanto estes podem se estabilizar sem promover ou propagar reações de oxidação. ${ }^{7}$

Domingos et al., utilizando o Rancimat à temperatura de $110^{\circ} \mathrm{C}$, estudaram a influência do BHA, BHT e TBHQ na estabilidade oxidativa do biodiesel B100 de óleo de soja. Verificaram que, em baixas concentrações, o BHA mostrou-se mais efetivo e, em concentrações altas, o TBHQ apresentou maior potencial estabilizador. ${ }^{8}$

O objetivo do trabalho foi apresentar uma análise do processo de oxidação do biodiesel através do acompanhamento da reação de oxidação em diferentes temperaturas, na presença ou ausência do antioxidante TBHQ.

\section{PARTE EXPERIMENTAL}

\section{Biodiesel}

As amostras de biodiesel B100, cedidas pelo Departamento de Química da UNICENTRO-PR, foram obtidas a partir da reação de transesterificação de óleo de soja por metanol, utilizando como catalisador o metóxido de sódio. ${ }^{9}$

\section{Análise físico-química do biodiesel B100}

Foi determinado, em triplicata, o ponto de fulgor (ABNT NBR 14598), índice de acidez (ABNT NBR 14448), massa específica a 20 ${ }^{\circ} \mathrm{C}$ (ABNT NBR 7148), estabilidade à oxidação a $110^{\circ} \mathrm{C}$ pelo método 
Rancimat ( EN 14112) e teor de metanol (EN 14110) segundo as metodologias recomendadas pela ANP. ${ }^{10}$

\section{Análise cromatográfica}

Foi utilizado um cromatógrafo modelo GC-17A com detector de ionização de chama, marca Shimadzu, com uma coluna DB1 (J\&W Scientific) $-100 \%$ polimetilsiloxano com $30 \mathrm{~m}$ de comprimento x $0,25 \mathrm{~mm}$ de diâmetro interno x $0,25 \mu \mathrm{m}$ de espessura de filme. ${ }^{9}$

\section{Antioxidante}

Foi utilizado $0,10 \%$ do antioxidante terc-butil-hidroquinona (TBHQ), grau P.A. que foi adicionado diretamente ao Biodiesel B100 antes da avaliação da estabilidade oxidativa. ${ }^{7,8}$

\section{Análise termogravimétrica}

A análise termogravimétrica do TBHQ foi realizada utilizando-se 10 mg de TBHQ no aparelho TG/DTA simultâneo Seiko 6300, com rampa de aquecimento de $25^{\circ} \mathrm{C}$ por minuto até atingir a temperatura de $500{ }^{\circ} \mathrm{C}$, permanecendo nessa temperatura por $5 \mathrm{~min}$, com fluxo de ar sintético de $100 \mathrm{~mL} / \mathrm{min}$.

\section{Avaliação da estabilidade oxidativa pelo teste de oxidação em estufa}

As amostras de biodiesel contendo $0,10 \%$ de TBHQ, bem como as amostras controle, foram levadas ao aquecimento em estufa a 30, 50 e $80{ }^{\circ} \mathrm{C}$ durante o tempo necessário para que todas as amostras atingissem o índice de peróxido máximo. ${ }^{11,12}$

\section{Índice de peróxido (I. P.)}

Durante o aquecimento em estufa foram coletadas, em triplicata, alíquotas para o acompanhamento do índice de peróxido, que foi determinado por titulação com tiossulfato de sódio pelo método AOAC Cd 8-53. ${ }^{13}$

\section{RESULTADOS E DISCUSSÃO}

A análise cromatográfica mostrou que o biodiesel utilizado consistia basicamente de ésteres metílicos com composição variando do C14 até C24. Os ésteres com composição química variando de $\mathrm{C} 8$ a $\mathrm{C} 14$, respectivamente, representaram $0,33 \% \mathrm{~m} / \mathrm{m}$ da amostra do biodiesel utilizado. O teor de éster metílico do ácido graxo C16:0 foi de $14,81 \%$ e dos ésteres metílicos dos ácidos graxos C18:0; C18:1 e C18:2 foi de 80,12\% $\mathrm{m} / \mathrm{m}$. Os ésteres formados por $\mathrm{C} 20$ a C22 representaram $2,04 \%_{\mathrm{m} / \mathrm{m}}$ da amostra do biodiesel. Estes ésteres somam $97,30 \%$ m/m das substâncias presentes no biodiesel B100 utilizado, estando, portanto, em acordo com a especificação da União Europeia, onde os teores de ácidos graxos livres, álcool, glicerina e água devem ser mínimos de modo que a pureza do biodiesel deve ser maior que $96,5 \% \mathrm{~m} / \mathrm{m} \cdot{ }^{14}$ Além disso, os teores de mono, di e triglicerídeos presentes somaram juntos $0,35 \%$ e o teor de glicerina livre observado foi de $0,002 \% \mathrm{~m} / \mathrm{m}$, valor bastante inferior ao da legislação brasileira, que prevê um teor máximo de glicerina livre de $0,02 \% \mathrm{~m} / \mathrm{m} \cdot{ }^{10,15}$

O biodiesel utilizado apresentou valores médios do teor de metanol de $0,07 \%$, ponto de fulgor de $165{ }^{\circ} \mathrm{C}$, massa específica a $20{ }^{\circ} \mathrm{C}$ de $880 \mathrm{~kg} / \mathrm{m}^{3}$, índice de acidez de $0,4 \mathrm{mg} \mathrm{KOH} / \mathrm{g}$, estando de acordo com a Resolução $07 .{ }^{10} \mathrm{O}$ período de indução a $110^{\circ} \mathrm{C}$ para a amostra contendo TBHQ foi de $9,78 \mathrm{~h}$ e $3,28 \mathrm{~h}$ para o controle, estando apenas este último valor fora das especificações estabelecidas pela mesma resolução.

A fim de avaliar a estabilidade oxidativa ou sua susceptibilidade à oxidação as amostras de biodiesel B100, contendo o TBHQ e o controle, foram sujeitas ao teste de oxidação acelerada em estufa. A temperatura máxima escolhida foi de $80^{\circ} \mathrm{C}$ e teve como base ensaios preliminares e a análise termogravimétrica do antioxidante utilizado, que apresentou um evento de decomposição térmica iniciando a $127^{\circ} \mathrm{C}$ e estendendo-se até $240^{\circ} \mathrm{C}$.

As respostas expressas em valores médios dos ensaios e do controle, para o índice de peróxido do biodiesel, estão apresentadas nas Tabelas 1, 2 e 3 . O tempo de duração total do ensaio para as amostras com TBHQ e controle, respectivamente, foram de 232 e $116 \mathrm{~d}$ para $30{ }^{\circ} \mathrm{C}, 62$ e 28 dias para $50{ }^{\circ} \mathrm{C}$ e de $6 \mathrm{~d}$ para ambos na temperatura de $80{ }^{\circ} \mathrm{C}$. Para efeito de comparação, foram considerados apenas os primeiros $92 ; 24$ e $5 \mathrm{~d}$ de ensaio, para 30,50 e $80^{\circ} \mathrm{C}$, respectivamente, pois estes foram os tempos necessários para que o controle atingisse $\mathrm{o}$ I.P. máximo, em cada temperatura considerada. Nos ensaios realizados a 30 e a $50{ }^{\circ} \mathrm{C}$, os tratamentos contendo TBHQ apresentaram os menores índices de peróxido Porém, para o ensaio realizado a $80^{\circ} \mathrm{C}$, a partir do $4^{\circ}$ dia, a amostra contendo o antioxidante apresentou maior I.P. quando comparado ao controle.

Tabela 1. Valores médios dos índices de peróxido obtidos a $30^{\circ} \mathrm{C}$

\begin{tabular}{ccccc}
\hline \multirow{2}{*}{ Tratamento } & \multicolumn{4}{c}{ Índice de peróxido (meq/kg amostra) } \\
\cline { 2 - 5 } & $14 \mathrm{~d}$ & $42 \mathrm{~d}$ & $59 \mathrm{~d}$ & $92 \mathrm{~d}$ \\
\hline TBHQ & 16,63 & 42,10 & 74,62 & 164,4 \\
Controle & 60,13 & 154,42 & 247,61 & 1297,94 \\
\hline
\end{tabular}

Tabela 2. Valores médios dos índices de peróxido obtidos a $50{ }^{\circ} \mathrm{C}$

\begin{tabular}{ccccc}
\hline \multirow{2}{*}{ Tratamento } & \multicolumn{4}{c}{ Índice de peróxido (meq/kg amostra) } \\
\cline { 2 - 5 } & $7 \mathrm{~d}$ & $14 \mathrm{~d}$ & $21 \mathrm{~d}$ & $24 \mathrm{~d}$ \\
\hline TBHQ & 22,44 & 28,64 & 41,00 & 55,16 \\
Controle & 114,45 & 405,87 & 944,42 & 1023,31 \\
\hline
\end{tabular}

Tabela 3. Valores médios dos índices de peróxido obtidos a $80^{\circ} \mathrm{C}$

\begin{tabular}{cccccc}
\hline \multirow{2}{*}{ Tratamento } & \multicolumn{5}{c}{ Índice de peróxido (meq/kg amostra) } \\
\cline { 2 - 6 } & $1 \mathrm{~d}$ & $2 \mathrm{~d}$ & $3 \mathrm{~d}$ & $4 \mathrm{~d}$ & $5 \mathrm{~d}$ \\
\hline TBHQ & 20,01 & 29,65 & 80,62 & 301,82 & 604,79 \\
Controle & 21,90 & 70,36 & 121,26 & 269,34 & 430,26 \\
\hline
\end{tabular}

As Figuras 1, 2 e 3 mostram a variação do índice de peróxido, expresso em miliequivalente por $\mathrm{kg}$ de amostra, pelo teste acelerado em estufa e o ponto de inflexão que corresponde ao período de indução, obtido pelo método de derivadas com interpolação de dados e alisamento por spline para as amostras de biodiesel B100 na presença e ausência do antioxidante TBHQ. ${ }^{16,17}$ Os testes foram realizados a 30,50 e $80{ }^{\circ} \mathrm{C}$, respectivamente, sendo que os pontos representam os dados experimentais de índice de peróxido.

Na Figura 1, o período de indução para a amostra controle foi de 75,32 d e para amostra contendo o antioxidante foi de 174,7 d.

Na Figura 2, o período de indução para a amostra controle foi de 18,6 dias e aquela contendo o antioxidante foi de 50,5 d. Portanto, foi observado que um aumento de $20^{\circ} \mathrm{C}$ reduziu em $246 \%$ o valor do período de indução para a amostra contendo o TBHQ.

Na Figura 3, o período de indução para o controle foi de 4,17 d e para amostra contendo o antioxidante foi de 4,25 d, uma diferença, 


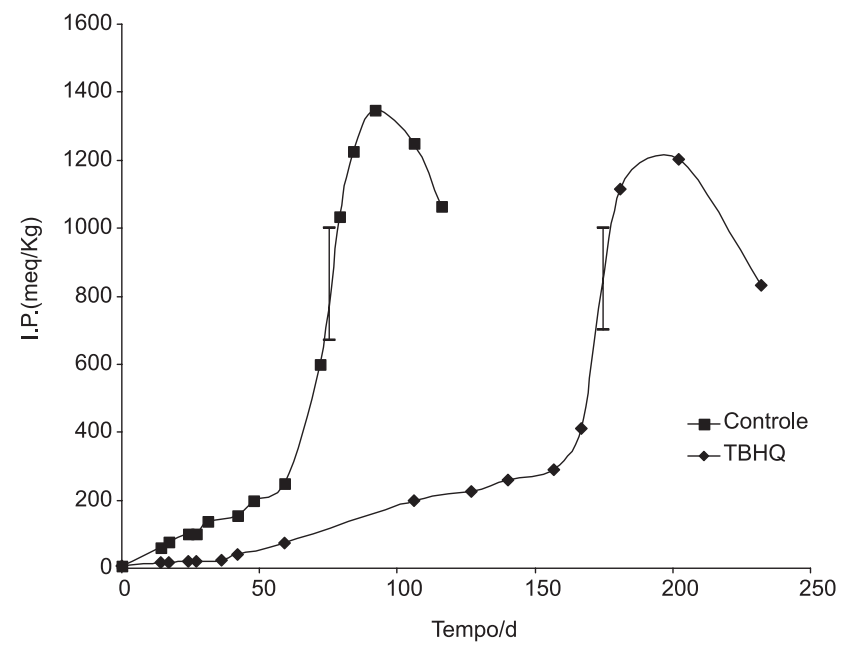

Figura 1. Índice de peróxido em função do tempo para a amostra contendo $T B H Q$ e o controle a $30^{\circ} \mathrm{C}$. Os pontos representam os dados experimentais e a reta mostra o ponto de inflexão

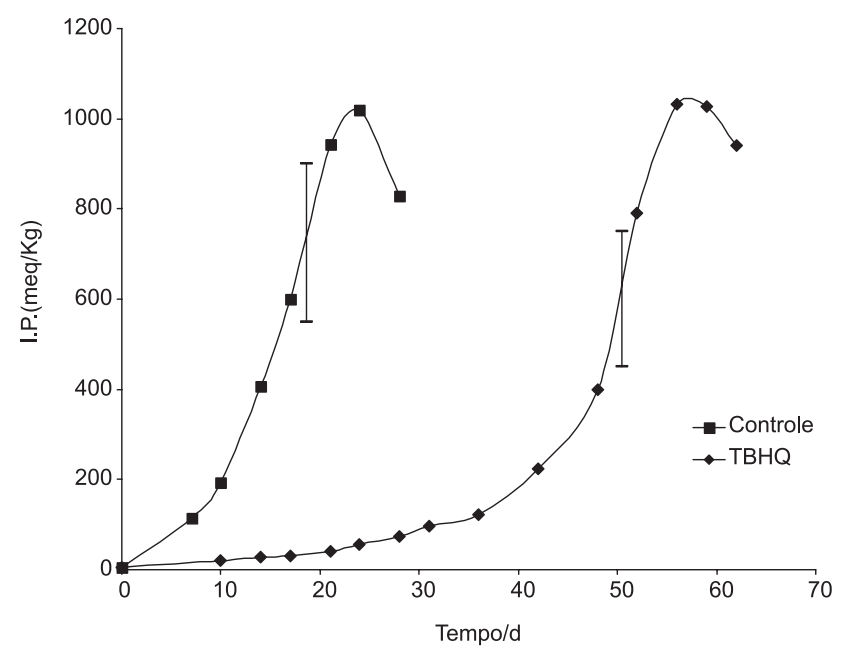

Figura 2. Índice de peróxido em função do tempo para a amostra contendo $T B H Q$ e o controle a $50^{\circ} \mathrm{C}$. Os pontos representam os dados experimentais e a reta mostra o ponto de inflexão

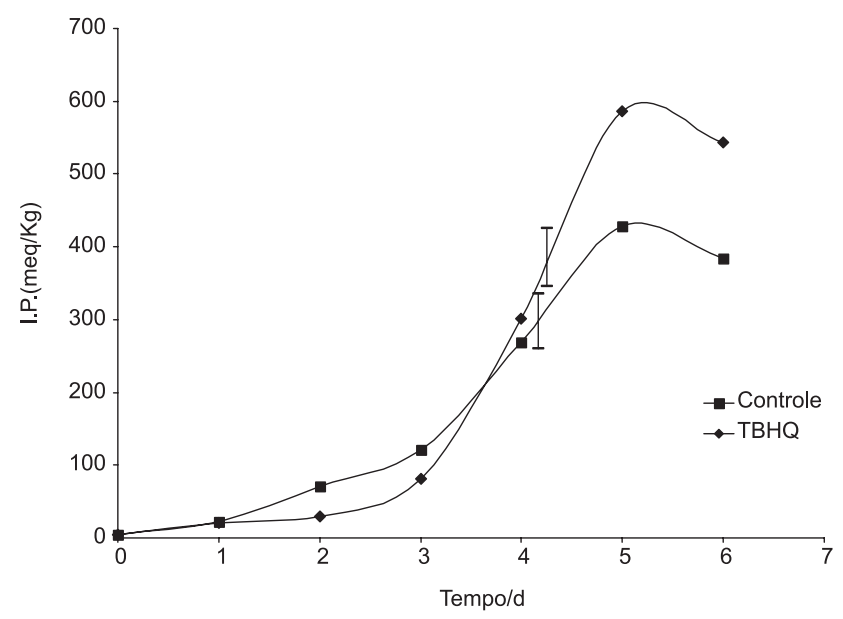

Figura 3. Índice de peróxido em função do tempo para a amostra contendo $T B H Q$ e o controle a $80^{\circ} \mathrm{C}$. Os pontos representam os dados experimentais e a reta mostra o ponto de inflexão entre eles, de apenas $2 \%$. Para todos os casos estudados o aumento de temperatura reduziu o intervalo observado entre o controle e a amostra contendo TBHQ.

Apesar do índice de peróxido não ser o mais indicado para o monitoramento da oxidação, devido à formação de produtos secundários, as Figuras 1, 2 e 3 bem como os estudos feitos por Ferrari e Souza e Borsato et al. mostraram que as curvas obtidas não apresentaram oscilações significativas. ${ }^{5,12}$ Pode-se observar pelo ponto de inflexão, que a iniciação, que corresponde ao período de indução que precede a propagação, foi retardada para o tratamento contendo o antioxidante sintético em todos os casos estudados.

As Figuras 1, 2 e 3 mostram ainda a propagação, uma fase exponencial, onde os radicais livres formados reagem com o substrato para dar origem a novos radicais livres e hidroperóxidos, e a terminação que corresponde à formação de produtos estáveis. ${ }^{7}$

A Figura 3 mostra que após $3,6 \mathrm{~d}$ de ensaio, a $80{ }^{\circ} \mathrm{C}$, o antioxidante utilizado deixa de apresentar efeito protetor contra a oxidação. Além disso, nesta temperatura, pode-se observar que os pontos de inflexão para os dois ensaios estão próximos. Isto indica que em temperaturas mais altas, o início da propagação da reação independe da presença ou não do antioxidante TBHQ.

De acordo com Frankel, por proporcionarem resultados mais rápidos, os métodos acelerados reduzem o tempo de trabalho e o consumo de reagentes. ${ }^{11}$ Ainda segundo ele, o método de estufa é o que apresenta menores limitações quando comparado com outros métodos sendo que, em temperaturas mais elevadas, os ensaios acelerados apresentam validade restrita.

A Tabela 4 mostra o tempo expresso em dias, para que o índice de peróxido máximo fosse atingido na amostra controle e naquela contendo o antioxidante TBHQ. Pode-se observar que a $30{ }^{\circ} \mathrm{C}$ o tempo em dias é 2,1 vezes maior quando se usa o TBHQ, 2,4 vezes maior a $50{ }^{\circ} \mathrm{C}$ e igual quando a temperatura de ensaio é de $80^{\circ} \mathrm{C}$. Os dados apresentados mostram uma ação protetora mais efetiva do antioxidante em temperaturas mais baixa.

Tabela 4. Tempo, em dias, para que o índice de peróxido máximo fosse atingido para a amostra contendo o antioxidante TBHQ e o controle a 30,50 e $80^{\circ} \mathrm{C}$

\begin{tabular}{cccc}
\hline \multirow{2}{*}{ Tratamento } & $30{ }^{\circ} \mathrm{C}$ & $50{ }^{\circ} \mathrm{C}$ & $80{ }^{\circ} \mathrm{C}$ \\
\cline { 2 - 4 } & \multicolumn{3}{c}{ Tempo (d) } \\
\hline TBHQ & 192 & 57 & 5 \\
Controle & 92 & 24 & 5 \\
\hline
\end{tabular}

As curvas apresentadas nas Figuras 1, 2 e 3 foram avaliadas com relação à cinética da reação do biodiesel B100 utilizado na presença e na ausência do antioxidante sintético TBHQ.

Quando não temos a possibilidade de conhecer o verdadeiro mecanismo da reação podemos propor uma equação de ordem $n$ mais geral para o ajustamento dos dados. ${ }^{5,18}$ Portanto, as curvas apresentadas nas Figuras 1, 2 e 3 foram avaliadas utilizando-se ordem de reação como sendo $\mathrm{n}=1$ e $0 \leq n<1$.

Com base na ordem de reação foram determinados os valores das constantes de velocidade, em d ${ }^{-1}$, nas três temperaturas consideradas.

A Tabela 5 apresenta os valores de n, k e $\mathrm{R}^{2}$ para cada situação, em cada temperatura de trabalho. A análise dos coeficientes de determinação total indica que a oxidação do biodiesel B100 na presença do antioxidante segue uma reação de primeira ordem e na ausência, uma ordem de reação $0 \leq n<1$.

Xin et al. atribuíram uma ordem de reação igual a 1 quando estudaram a estabilidade de biodiesel de óleo de girassol contendo diferentes concentrações do antioxidante sintético galato de propila. ${ }^{19}$ 
Tabela 5. Ordem da reação $\mathrm{n}$ e as constantes de velocidade k para as três temperaturas utilizadas

\begin{tabular}{|c|c|c|c|c|c|c|c|c|c|}
\hline & \multicolumn{3}{|c|}{$30^{\circ} \mathrm{C}$} & \multicolumn{3}{|c|}{$50^{\circ} \mathrm{C}$} & \multicolumn{3}{|c|}{$80^{\circ} \mathrm{C}$} \\
\hline & $\mathrm{n}$ & $\mathrm{k}$ & $\mathrm{R}^{2}$ & $\mathrm{n}$ & $\mathrm{k}$ & $\mathrm{R}^{2}$ & $\mathrm{n}$ & $\mathrm{k}$ & $\mathrm{R}^{2}$ \\
\hline \multirow{2}{*}{ TBHQ } & 0,3464 & 0,3988 & 0,9601 & 0,6048 & 0,5661 & 0,9142 & 0,5861 & 5,9564 & 0,9146 \\
\hline & 1,0000 & 0,0104 & 0,9594 & 1,0000 & 0,0383 & 0,9889 & 1,0000 & 0,3943 & 0,9853 \\
\hline \multirow{2}{*}{ Controle } & 0,4238 & 1,0608 & 0,9438 & 0,4841 & 3,0349 & 0,9942 & 0,4853 & 8,1901 & 0,9936 \\
\hline & 1,0000 & 0,0212 & 0,8942 & 1,0000 & 0,0918 & 0,8853 & 1,0000 & 0,3581 & 0,9469 \\
\hline
\end{tabular}

Como esperado, os menores valores de $k$ foram obtidos para os tratamentos contendo TBHQ.

Os valores de $\mathrm{n}$ para a amostra controle apresentaram uma estimativa do desvio padrão de apenas 0,029 com um erro padrão igual a 0,0166 em relação à média. Em adição, o coeficiente de variação, que dá uma ideia da precisão do experimento, foi de apenas $6 \%$ que é um valor considerado baixo. ${ }^{20}$ Ainda para a amostra controle, quando se comparam os valores de $\mathrm{n}$ em relação à média, a diferença foi menor que $5 \%(4,24$ e $4,50 \%)$ para as temperaturas de 50 e $80^{\circ} \mathrm{C}$ porém, para o ensaio a $30^{\circ} \mathrm{C}$, foi observada uma diferença maior $(8,74 \%)$. Reações de oxidação de biodiesel B100, obtido de óleo de vegetal, ocorrem à temperatura ambiente e, segundo Levenspiel, reações deste tipo são muito sensíveis à variação de temperatura. ${ }^{5,21}$ De fato, a diferença entre as ordens das reações é maior entre as temperaturas de 30 e $50{ }^{\circ} \mathrm{C}$ e 30 e $80{ }^{\circ} \mathrm{C}$ do que entre 50 e $80{ }^{\circ} \mathrm{C}$.

Portanto, os dados da Tabela 5 mostram que o mecanismo da reação de oxidação do biodiesel não se alterou significativamente com o aumento da temperatura de ensaio, tanto para a amostra controle quanto para aquela contendo TBHQ. Além disso, o alto coeficiente de correlação linear obtido sugere a aplicação da Equação de Arrhenius para obtenção da energia de ativação.

As Figuras 4 e 5 mostram a relação entre o logaritmo natural das constantes de velocidade com $\mathrm{T}^{1}$.

De acordo com Levenspiel, reações com valores variando entre 40 e $400 \mathrm{~kJ} \mathrm{~mol}^{-1}$ são consideradas de baixa e alta energia de ativação, respectivamente. ${ }^{21}$

A energia de ativação calculada para o controle foi $\mathrm{Ea}=36,11 \mathrm{~kJ}$ $\mathrm{mol}^{-1}$ e para o ensaio contendo TBHQ foi $\mathrm{Ea}=65,08 \mathrm{~kJ} \mathrm{~mol}^{-1}$. Estes dados estão em acordo com Xin et al., que obtiveram uma energia de ativação de $97,02 \mathrm{~kJ} \mathrm{~mol}^{-1}$ quando estudaram a cinética da reação em biodiesel de óleo de girassol utilizando o galato de propila como antioxidante. ${ }^{19}$ Portanto, o biodiesel empregado apresentou pouca

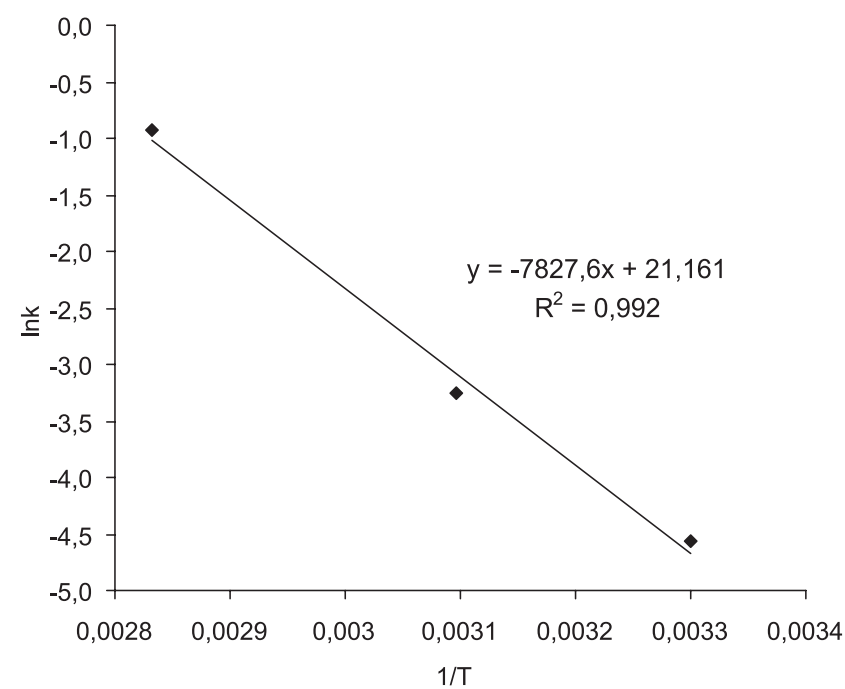

Figura 4. Logaritmo natural das constantes de velocidade com o inverso da temperatura, em Kelvin, para a amostra com $T B H Q$

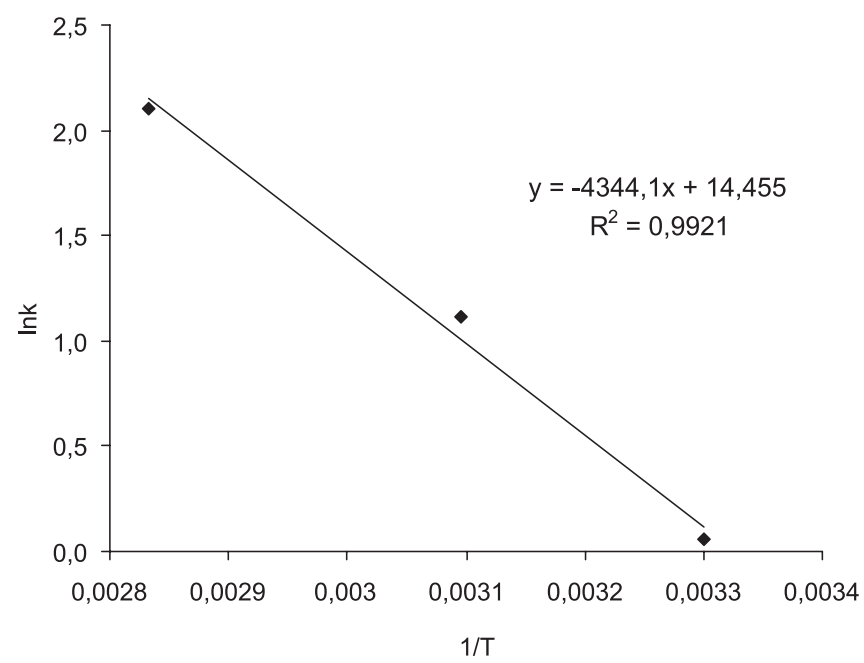

Figura 5. Logaritmo natural das constantes de velocidade com o inverso da temperatura, em Kelvin, para a amostra controle

estabilidade química porque os valores obtidos da energia de ativação foram baixos. Como a energia de ativação em presença do TBHQ foi maior que o controle, houve um aumento da estabilidade na presença do antioxidante sintético.

Hasenhuettl e Wan encontraram uma alta correlação linear entre o logaritmo natural do período de indução em função da temperatura quando estudaram a estabilidade oxidativa de seis diferentes tipos de óleos vegetais sem o uso de antioxidantes, utilizando o teste de oxidação acelerada pelo método Rancimat. ${ }^{22}$ Porém, os mesmos autores reportaram que a extrapolação para temperaturas menores, como aquelas verificadas durante o armazenamento, é questionável. De fato, de acordo com Frankel, os métodos de estabilidade acelerada apresentam validade restrita, pois à medida que se submete a amostra ao aquecimento, à luz ou ao contato com metais se muda o mecanismo da reação de oxidação, sendo que os testes efetuados em temperaturas mais próximas da temperatura ambiente se aproximam da estocagem real. ${ }^{11}$

A Figura 6 mostra a temperatura em função do logaritmo natural do período de indução para o biodiesel B100 de óleo de soja estabilizado com TBHQ e controle.

Foi observada uma alta correlação linear entre os tratamentos contendo o antioxidante e o controle, obtendo-se valores superiores a 99\%. Portanto, as retas da Figura 6 são úteis para a correção da temperatura e sugerem a possibilidade de extrapolar os dados para se obter o tempo de estocagem em temperaturas mais baixas, em condições mais realistas de armazenamento. Dessa maneira, foi possível verificar que o biodiesel contendo TBHQ pode apresentar um tempo de estocagem, a $25^{\circ} \mathrm{C}$, três vezes maior que o tempo verificado para o controle, mostrando a eficácia do antioxidante utilizado. Portanto, de acordo com a Figura 6, a presença de $0,1 \%$ de TBHQ pode garantir 283 dias de armazenamento sem uma degradação evidente, o que pode ser considerado um intervalo de tempo realista para a vida comercial do biodiesel B100 obtido de óleo de soja. 


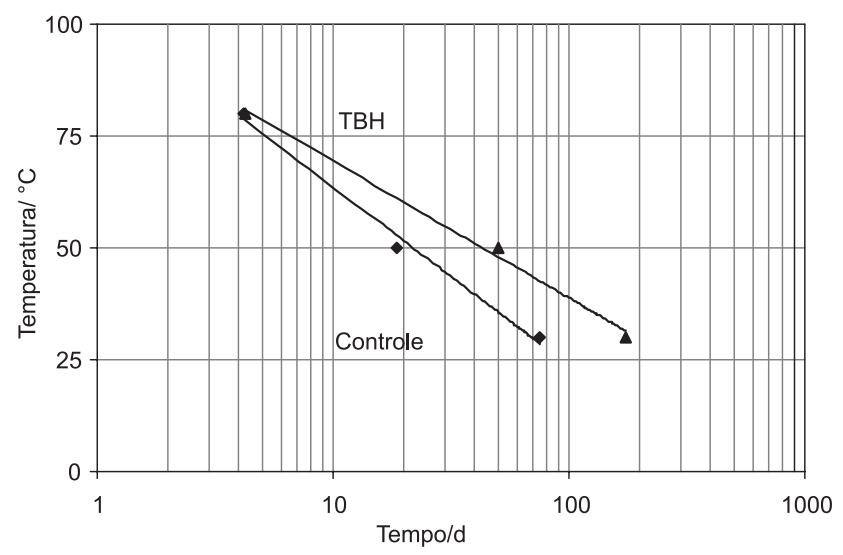

Figura 6. Relação entre a temperatura $\left({ }^{\circ} \mathrm{C}\right)$ e o logaritmo natural do período de indução do biodiesel de soja estabilizado com antioxidantes e controle, em dias

\section{CONCLUSÕES}

O período de indução que precede a propagação do processo oxidativo do biodiesel B100, quando comparado com o controle, foi retardado para o ensaio contendo o antioxidante.

À medida que a temperatura de ensaio aumenta, diminui a diferença entre o período de indução do controle e da amostra contendo o antioxidante, indicando que o aumento da temperatura diminui o efeito protetor do TBHQ.

A energia de ativação determinada para a reação em estudo foi baixa e o uso do TBHQ no biodiesel foi mais eficiente quando a temperatura de estocagem foi mais próxima da ambiente. Além disso, como a energia de ativação em presença do TBHQ foi maior que o controle, houve um aumento da estabilidade com a adição do antioxidante.

Através dos resultados obtidos, nas temperaturas utilizadas, verificou-se que o ensaio contendo $0,1 \%$ de TBHQ se mostrou eficiente para aumentar a estabilidade oxidativa de biodiesel B100 de óleo de soja.

\section{AGRADECIMENTOS}

À Fundação Araucária pelo suporte financeiro, à UEL e ao CNPq pela concessão de bolsas.

\section{REFERÊNCIAS}

1. Knothe G.; Fuel Process Technol. 2007, 88, 669.

2. Monyem, A.; van Gerpen, J. H.; Biomass Bioenerg. 2001, 20, 317.

3. Meher, L. C.; Vidya Sagar, D.; Naik, S. N.; Renew. Sust. Energy Rev. 2006, 10, 248.

4. Borsato, D.; Moreira, I.; Pinto, J. P.; Moreira, M. B.; Nobrega, M. M.; Constantino, L. V.; Acta Sci. 2010, 32, 187.

5. Borsato, D.; Dall'Antonia, L. H.; Guedes, C. L. B.; Maia, E. C. R.; Freitas, H. R.; Moreira, I.; Spacino, K. R.; Quim. Nova 2010, 33, 1726.

6. McCormick, R. L.; Ratcliff, M. M.; Lawrence, R.; Fuel Process Technol. 2007, 88, 651 .

7. Ramalho, V. C.; Jorge, N.; Quim. Nova 2006, 29, 755.

8. Domingos, A. K.; Saad, E. B.; Vechiatto, W. W. D.; Wilhelm, H. M.; Ramos, L. P.; J. Braz. Chem. Soc. 2007, 18, 416.

9. Borsato, D.; Guedes, C. L. B.; Moreira, I.; Pinto, J. P.; Dias, G. H.; Spacino, K. R.; Semina 2010, 31, 3.

10. Brasil; Resolução $n^{o} 7$, de 19/03/2008 - ANP, Agência Nacional do Petróleo, Gás Natural e Biocombustíveis. Estabelece a especificação para a comercialização de biodiesel que poderá ser adicionado ao óleo diesel. Diário Oficial da União de 20/03/2008.

11. Frankel, E. N.; Trends Food Sci. Technol.1993, 4, 220.

12. Ferrari, R. A.; Souza, W. L.; Quim. Nova 2009, 32, 106.

13. A.O.C.S - American Oil Chemist's Society; Official Methods and recommended praticces of the American Oil Chemists Society, $5^{\text {th }}$ ed., Champaign: USA, 1998.

14. Karaosmanoglu, F.; Becker, U. G.; Energ. Source 1996, 18, 637.

15. Lôbo, I. P.; Ferreira, S. L. C.; Cruz, R. S.; Quim. Nova 2009, 32, 1596.

16. Greville, T. N. E.; Theory and aplications of spline functions, $1^{\text {st }}$ ed., Academic Press: New York, 1969.

17. Boor, C.; A practical guide do Spline, $1^{\text {st }}$ ed., Springer-Verlag: New York, 1978.

18. Petriella, C.; Resnik, S. L.; Lozano, R. D.; Chirife, J.; J. Food Sci. 1985, 50,622 .

19. Xin, J.; Imahara, H.; Saka, S.; Fuel 2009, 88, 282.

20. Gomes, F. P.; Curso de estatística experimental, $11^{\mathrm{a}}$ ed., Livraria Nobel: Piracicaba, 1985.

21. Levenspiel, O.; Chemical reaction engineering, $3^{\text {rd }}$ ed., John Wiley and Sons Inc: New York, 1999.

22. Hasenhuettl, G. L.; Wan, P. J.; J. Am. Oil Chem. Soc. 1992, 69, 525. 